Pacific Journal of Mathematics

A BOUNDARY FOR THE ALGEBRAS OF BOUNDED
HOLOMORPHIC FUNCTIONS 


\title{
A BOUNDARY FOR THE ALGEBRAS OF BOUNDED HOLOMORPHIC FUNCTIONS
}

\author{
DoNG S. KIM
}

Let $(X, A)$ be a ringed space and let $D$ be a domain in $X$. Let $B=B(D)=\left\{f \in A(D) ;\|f\|_{D}<\infty\right\}$. A minimal boundary for $B$ is defined as a unique smallest subset of $\bar{D}$ such that every function in $B$ attains its supremum near the set. The followings are shown: If $X$ is locally compact, $D$ is relatively compact, and $B$ separates the points of $D$ then there exists a minimal boundary. Under the same assumptions, the natural projection of the Silov boundary $\partial_{\hat{B}}$ into $X$ is the minimal boundary. If $A$ is a maximum modulus algebra and the set of frontier points for $A$ is the minimal boundary, then any holomorphic function which is bounded near the minimal boundary must be bounded. Finally, if $D$ is the spectrum of $B$ (with the compact open topology), then the topological boundary of $D$ is the set of frontier points for $B$.

Introduction. Let $(X, A)$ be a ringed space; a subsheaf of rings with identity of the sheaf of germs of continuous functions on a Hausdorff space $X$. Let $\Gamma(U, A)$ be the set of all sections of $A$ over $U, U$ is an open subset of $X$. Let $A(U)=\{f \in C(U): f(x)=\phi(x)(x)=$ $\left.{ }_{x} f(x), x \in U\right\}$, where $\phi \in \Gamma(U, A)$ and ${ }_{x} f$ is the germ of $f$ at $x$. A function $f$ in $A(U)$ is called $A$-holomorphic or holomorphic. Let $B(U)=$ $\{f \in A(U): f$ is bounded on $U\}$. Then $B(U)$ is an algebra (over $C$ ) with identity.

Let $D$ be an open subset of $X$ and let $\bar{D}$ be the closure of $D$ in $X$. For $\Delta \subset \bar{D}$ let $N(\Delta)$ be the filter base of open neighborhoods of $\Delta$ in $X$ and denote $N_{0}(\Delta)$ be the trace of $N(\Delta)$ on $D$.

Definition. For $f \in A(D)$, define $\operatorname{cl} t_{f}(\Delta)=\left\{\bigcap \operatorname{cl} f(W): W \in N_{0}(\Delta)\right\}$, where $\operatorname{cl} f(W)$ is the closure of $f(W)$ in the Riemann sphere $C \cup(\infty\}$, the cluster set of $f$ at $\Delta$, and write $\mathrm{cl} t_{f}(x)$ for $\mathrm{cl} t_{f}(\{x\})$. Define $M_{f}(\Delta)=\sup \left|\operatorname{cl} t_{f}(\Delta)\right| \in[0, \infty]$, and write $M_{f}(x)$ for $M_{f}(\{x\})$.

Let $B=B(D)$. Denote $B_{s}$ for $B$ with the topology of supremum norm on $D$ and $B_{c}$ for $B$ with the topology of uniform convergence on compact subsets of $D$ (c.o. topology). Then $B_{s}$ is a Banach algebra. Let $S\left(B_{s}\right)$ be the space of nonzero complex homomorphisms of $B_{s}$ onto $C$ and $S\left(B_{c}\right)$ be the space of nonzero continuous complex homomorphisms of $B_{c}$ onto $C$. Then $S\left(B_{s}\right) \supset S\left(B_{c}\right)$, for, if $h \in S\left(B_{c}\right)$ then there exists a compact subset $K_{h}$ of $D$ such that $|h(f)| \leqq\|f\|_{k_{h}}$ for all $f \in B$, which implies $|h(f)| \leqq\|f\|_{D}$ for all $f \in B$, so that $h \in S\left(B_{s}\right)$. Endow $S\left(B_{s}\right)$ with the weakest topology for which each $\hat{f}$ is continuous, 
where $\hat{f}$ is the Gelfand representation of $f$ on $S\left(B_{s}\right)$ such that $\hat{f}(h)=$ $h(f)$ for all $h \in S\left(B_{s}\right)$. Then $S\left(B_{s}\right)$ is compact. Equip $S\left(B_{c}\right)$ with the relative topology of $S\left(B_{s}\right)$. For $x \in D$ define $h_{x}(f)=f(x)$ for all $f \in B$ then $h_{x} \in S\left(B_{s}\right)$, moreover $h_{x} \in S\left(B_{c}\right)$, since $\left|h_{x}(f)\right|=|f(x)| \leqq\|f\|_{K}$ for all $f \in B$, where $K$ is a compact subset containing $\{x\}$. Now if $B$ separates the points of $D$ then it separates strongly the points of $D$ (in the sense of [8]), since $B$ contains constant functions. If $D$ is locally compact and $B$ separates the points then the natural embedding $\rho$ of $D$ into $S\left(B_{s}\right)$ is a homeomorphism (See Cor. 3.2.5 of Rickart [8]). Henceforth, we denote $\rho$ for this homeomorphism. Let $\pi$ be a continuous mapping from $S\left(B_{s}\right)$ into $X$ such that $\pi \mid \rho D$ is the inverse mapping of $\rho$, so that $\pi \mid \rho D$ is a homeomorphism of $\rho D$ onto $D$.

The prototype of these phenomena is the following: Let $D$ be a relatively compact domain in $C^{n}$ and $B=B(D)$. Set $S=S\left(B_{s}\right)$. With the coordinate function $z_{1}, z_{2}, \cdots, z_{n}$ in $B$, define $\pi: S \rightarrow C^{n}$ by $\pi(h)=\left(\widehat{z}_{1}(h), \cdots, \widehat{z}_{n}(h)\right), h \in S\left(\pi(S)\right.$ is the joint spectrum of $z_{1}, z_{2}, \cdots, z_{n}$. Then $\pi$ is continuous and it is a homeomorphism on $\rho D$. Moreover $\pi s\left({ }_{c} B\right) \subset D$ and $\pi S \supset \bar{D}$.

\section{A minimal boundary.}

\section{Proposition 1.}

(i ) $M_{f}(\Delta)=\lim _{N_{0}(\Delta)} \sup \left\{|f(W)|: W \in N_{0}(\Delta)\right\}$, where $\Delta \subset \bar{D}$. For $x \in D, M_{f}(x)=f(x) . \quad\|f\|=\sup _{x \in D}|f(x)|=M_{f}(D)=M_{f}(\bar{D})$.

(ii) The function $M_{f}(\cdot): \bar{D} \rightarrow[0, \infty]$ is upper semi-continuous.

(iii) For a closed subset $\Delta \subset \bar{D}$, there exists a point $p \in \Delta$ such that $M_{f}(\Delta)=M_{f}(p)$.

(iv) $M_{f g}(\Delta) \leqq M_{f}(\Delta) \cdot M_{g}(\Delta)$, where $\Delta \subset \bar{D}$.

Proof. For (i), (ii), and (iii), see Quigley [5]. (iv) is trivial.

Definition 2. Let $H \subset A(D)$. We call a subset $\Gamma$ of $\bar{D}$ an $H$-set if $\Gamma$ is closed in $\bar{D}$ and $\|f\|=M_{f}(D)=M_{f}(\Gamma)$ for all $f \in H$. An $H$-set is minimal if it properly contains no $H$-set. Denote $\Gamma_{H}$ for a minimal $H$-set.

If $H=B=B(D), \Gamma_{B}$ is a minimal $B$-set.

Proposition 2. If $D$ is relatively compact then a minimal $H$-set exists for every $H \subset A(D)$.

Proof. See Quigley [5].

Proposition 3. Let $X$ be locally compact and $B$ separate the points of $D$. Let $\pi$ be a continuous mapping from $S\left(B_{s}\right)$ into $X$ such that $\pi \circ \rho$ is the identity mapping on $D$. Let $\mathrm{cl} \rho D$ be the closure of $\rho D$ in $S\left(B_{s}\right)$. Then $\pi(\operatorname{cl} \rho D)=\bar{D}$ and $\pi(\operatorname{cl} \rho D-\rho D)=\bar{D}-D$. 
Proof. Since cl $\rho D$ is compact and $\pi(\operatorname{cl} \rho D) \supseteqq D, \pi(\operatorname{cl} \rho D) \supseteqq \bar{D}$. Let $h \in \operatorname{cl} \rho D$ then for any net $\left\{h_{n}\right\} \subset \rho D$ which converges to $h,\left\{\pi\left(h_{n}\right)\right\}$ converges to $\pi(h)$, since $\pi$ is continuous. Since $\left\{\pi\left(h_{n}\right)\right\} \subset D, \pi(h) \in \bar{D}$. So $\pi(\operatorname{cl} \rho D) \subseteq \bar{D}$. Hence $\pi(\operatorname{cl} \rho D)=\bar{D}$.

Let $h \in \operatorname{cl} \rho D-\rho D$ and assume that $\pi(h) \in D$. Take any $f \in B$. Since $f$ is continuous, we may choose, for arbitrary $\varepsilon^{\prime}>0$, a neighborhood $U$ of $\pi(h) ; U=\left\{x \in D:\left|f_{i}(x)-f_{i}(\pi(h))\right|<\varepsilon, i=1,2, \cdots, n\right\}$, such that $y \in U$ implies $|f(y)-f(\pi(h))|<\varepsilon^{\prime}$. Again, since $\hat{f}$ is continuous on $S\left(B_{8}\right)$ and $h \in \operatorname{cl} \rho D$, there is $y_{0} \in D$ with $\rho\left(y_{0}\right) \in N=\{\rho \in$ $\left.S\left(B_{s}\right):\left|\hat{f}_{i}(\varphi)-\hat{f}_{i}(h)\right|<\varepsilon, i=1,2, \cdots, n\right\}$ such that $\left|\hat{f}(h)-\hat{f}\left(\rho\left(y_{0}\right)\right)\right|<\varepsilon^{\prime}$. Note that $y_{0} \in U=\pi \mid \rho D(N)$, so $\left|f\left(y_{0}\right)-f(\pi(h))\right|<\varepsilon^{\prime}$. Also $f\left(y_{0}\right)=$ $\hat{f}\left(\rho\left(y_{0}\right)\right)$ and $f(\pi(h))=\hat{f}\left(\rho(\pi(h))\right.$, so it follows that $|\hat{f}(h)-\hat{f}(\rho(\pi(h)))|<2 \varepsilon^{\prime}$. Since $\varepsilon^{\prime}$ is arbitrary, we have $\hat{f}(h)=\hat{f}(\rho(\pi(h)))$ foy every $f \in B$. Hence $h=\rho(\pi(h)) \in \rho D$, which is absurd. Hence $\pi(\operatorname{cl} \rho D-\rho D)=\bar{D}-D$.

THeORem 1. Let $X$ be locally compact and $D$ be relatively compact in $X$. If $B(D)$ separates the points of $D$, then the minimal $B$ set $\Gamma_{B}$ is unique.

Proof. Let $\Gamma_{1}$ and $\Gamma_{2}$ be minimal $B$-sets, and let $p \in \Gamma_{1}$ be an arbitrary point of $\Gamma_{1}$. We will show that every neighborhood of $p$ intersects $\Gamma_{2}$ so that $p \in \Gamma_{2}$. So $\Gamma_{1} \subset \Gamma_{2}$. The same argument shows that $\Gamma_{2} \subset \Gamma_{1}$.

Let $p \in \Gamma_{1}$. Let $W$ be any neighborhood of $p$ in $\bar{D}$ and let $\varphi \in$ cl $\rho D$ such that $\pi(\varphi)=p$. Take a neighborhood $N$ of $\varphi$ in $S\left(B_{s}\right)=S$ such that $N \subset \pi^{-1}(W) ; N=\left\{h \in S:\left|\hat{f}_{i}(h)-\hat{f}_{i}(\varphi)\right|<\varepsilon, i=1,2, \cdots, n\right\}$. Put $U=\left\{x \in D:\left|f_{i}(x)-a_{i}\right|<\varepsilon, i=1,2, \cdots, n\right\}$, where $a_{i}=\hat{f}_{i}(\varphi)$. Then $U=\pi(N) \cap D \subset \pi(N)$. Let $V=\left\{x \in \bar{D}: M_{f_{i}-a_{i}}(x)<\varepsilon / 2, i=1,2, \cdots, n\right\}$. Since $M_{f_{i}-a_{i}}(x)=\left|f_{i}(x)-a_{i}\right|$ for $x \in D, V \cap D=U$. And, since $M_{f_{i}-a_{i}}$ is upper semicontinuous, $V$ is open in $\bar{D}$ and it is easy to see that $M_{f_{i}-a_{i}}(p)=0$, so $V$ is an open neighborhood of $p$. Note that $M_{f_{i}}(p)=$ $\left|a_{i}\right|$. Now, since $M_{f_{i}-a_{i}}(x)<\varepsilon / 2$ in $V$, we may choose a neighborhood $G$ of $p$ in $\bar{D}$ such that $\left|\left(f_{i}-a_{i}\right)(x)\right|<\varepsilon$ for all $x \in G \cap D$ and $G \subset \pi N$. Then $V \subset G \subseteq \pi N \subset W$.

Since $\Gamma_{1}-V$ is closed in $\bar{D}$ and it is a proper subset of $\Gamma_{1}$, it is not a $B$-set. Hence there exists $g \in B(D)$ such that $M_{g}\left(\Gamma_{1}-V\right)<$ $M_{g}\left(\Gamma_{1}\right)=\|g\|$. So $M_{g}\left(\Gamma_{1}-V\right)\|g\|^{-1}<1$. Choose $m$ large enough such that $\left\{M_{g}\left(\Gamma_{1}-V\right)\|g\|^{-1}\right\}^{m}<\varepsilon\left(1+\sum_{1}^{n}\left\|f_{i}-a_{i}\right\|\right)^{-1}=\delta$, and set $f=g^{m}$. Then $M_{f}\left(\Gamma_{1}-V\right)=M_{g^{m}}(\Gamma-V) \leqq\left\{M_{g}(\Gamma-V)\right\}^{m}<\delta\|g\|^{m}=$ $\delta\|f\|$. If $x \in V$ then $M_{f_{i}-a_{i}}(x)<\varepsilon / 2$ so that

$$
M_{f_{i-2}} M_{f}(x)=M_{f_{i}-a_{i}}(x) M_{f}(x)<\frac{\varepsilon}{2} M_{f}(\bar{D})=\frac{\varepsilon}{2}\|f\| .
$$

If $x \in \Gamma_{1}-V$ then $M_{f}(x) \leqq M_{f}\left(\Gamma_{1}-V\right)<\delta\|f\|$, so that again 


$$
M_{f_{i}-a_{i}} M_{f}(x)<\frac{\varepsilon}{2}\|f\|
$$

Since $\Gamma_{1}$ is a $B$-set it follows that $M_{f_{i}-a_{i}} M_{f}(\bar{D})<(\varepsilon / 2) M_{f}(\bar{D})=(\varepsilon / 2)\|f\|$. Let $q$ be any point of $\Gamma_{2}$ with $M_{f}(q)=M_{f}(\bar{D})=M_{f}(D)=\|f\|$. Then $M_{f_{i}-a_{i}}(q) M_{f}(q)<(\varepsilon / 2)\|f\|$. Hence $M_{f_{i}-a_{i}}(q)<\varepsilon / 2$ and this is true for all $i=1,2, \cdots, n$. Thus $q \in V$, so $V \cap \Gamma_{2} \neq \varnothing$. Hence $W \cap \Gamma_{2} \neq \phi$. Since $\Gamma_{2}$ is closed, $p \in \Gamma_{2}$. The proof is complete.

We call the unique minimal $B$-set the minimal boundary for $B$.

Note. Let $\Gamma_{B}$ be a minimal boundary for $B$ then $x \in \Gamma_{B}$ if and only if for every neighborhood $U$ of $x$ there exists $f \in B$ such that $\|f\|=M_{f}(U)>M_{f}(\bar{D}-U)$.

THEOREM 2. Let $X$ be locally compact and $D$ be relatively compact in $X$. We assume that $B$ separates the points of $D$. Then $\pi \hat{\partial} \hat{B}$ is a minimal boundary.

Proof. Since $M_{f}(\bar{D})=\|f\|_{D}=\|\hat{f}\| \rho_{D}=\|\hat{f}\|_{S}$ for all $f \in B$, we have $\partial_{\hat{B}} \subset \operatorname{cl} \rho D$. Let $x \in \pi \partial_{\hat{B}}$ then there exists $h \in \partial_{\hat{B}}$ such that $x=\pi h$. Now, $h \in \partial_{\hat{B}}$ implies that for arbitrary neighborhood $N$ of $h$ in $S=S\left(B_{s}\right)$ there exists $\hat{f} \in \hat{B}$ such that $\|\hat{f}\|_{S}=\|\hat{f}\|_{N}>\|\hat{f}\|_{S-N}$. Since $S-N \supset$ $\rho D-N \cap \rho D$, we have $\|\hat{f}\|_{S-N} \geqq\|\hat{f}\|_{\rho_{D-N} \rho_{D}}$. So $\|\hat{f}\|_{\rho D}=\|\hat{f}\|_{S}>$ $\|\hat{f}\|_{S-N} \geqq\|\hat{f}\|_{\rho D-N \cap \rho_{D}}$. Hence it follows that $\|\hat{f}\|_{\rho_{D}}=\|\hat{f}\|_{N \cap \rho D}>$ $\|\hat{f}\|_{\rho D-N \cap \rho_{D}}$. This is equivalent to $\|f\|_{D}=\|f\|_{\pi\left(N \cap \rho_{D}\right)}>\|f\|_{D-\pi(N \cap \rho D)}$. Since $\pi(N \cap \rho D)$ is a trace of a neighborhood of $x=\pi h$ on $D$ and a trace of any neighborhood of $x$ on $D$ can be written as such a form, $x=\pi h$ belongs to a minimal boundary $\Gamma_{B}$. So $\pi \partial_{\hat{B}} \subset \Gamma_{B}$. On the other hand, if $W$ is any open set containing $\pi \partial_{\hat{B}}$, then by the continuity of $\pi$, there exists an open set $G$ in $S$ containing $\partial_{\hat{B}}$ such that $\pi(G) \leqq W$ and hence $\pi(G \cap \rho D) \subseteq W \cap D$. For any $f \in B$, we have

$$
\|f\|_{W \cap D} \geqq\|\hat{f}\|_{G \cap \rho D}=\|\hat{f}\|_{G \cap c l \rho D}=\|\hat{f}\|_{\partial \hat{B}}=\|f\|_{D} \text {. }
$$

If follows that $M_{f}\left(\pi \partial_{\hat{B}}\right)=\|f\|_{D}$ for all $f \in B$. Since $\pi \partial_{\hat{B}}$ is closed, it is a $B$-set. Thus $\pi \partial_{\hat{B}}$ is a minimal boundary.

For instance: Let $D$ be the unit open disc in $C$ and let $B(D)=H^{\infty}$. Define a natural continuous mapping $\pi$ of $S$ into the closed unit disc $\bar{D}$ by $\pi(h)=h(z), h \in S$ and $z$ is the coordinate function. Then the minimal boundary $\Gamma_{B}$ is the unit circle and the Silov boundary $\partial_{\hat{B}}$ on $S$ is a proper closed subset of $\operatorname{cl} \rho D-\rho D$ which is totally disconnected. The image of $\partial_{\hat{B}}$ under $\pi$ is the unit circle.

Next, we have a question that whether a function $f$ with $M_{f}\left(\Gamma_{B}\right)<\infty$ is bounded. 
Proposition 4. Suppose $A=A(D)$ and $B=B(D)$ have the unique minimal boundaries $\Gamma_{A}$ and $\Gamma_{B}$ respectively. If $\Gamma_{A} \neq \Gamma_{B}$ then there exists a function $f \in A$ which is bounded near $\Gamma_{B}$ (i.e., $M_{f}\left(\Gamma_{B}\right)<\infty$ ), but not in $B$.

Proof. In general, $\Gamma_{A} \supset \Gamma_{B}$. Take $x \in \Gamma_{A}-\Gamma_{B}$ and choose a neighborhood $U$ of $x$ in $\bar{D}$ such that $M_{f}(U)=\|f\|>M_{f}(\bar{D}-U)$ and $U \cap \Gamma_{B}=\phi$. Then $M_{f}\left(\Gamma_{B}\right)<\infty$ but $f \notin B$.

Definition. A point $x \in \bar{D}$ is a frontier point of $D$ for $H \subset A(D)$ if for each compact subset $K$ of $D$ with $x \notin K$ there exists $f \in H$ such that $M_{f}(x)>\|f\|_{K}$. Let $F_{H}$ be the set of all frontier points of $D$ for $H$. Denote $F_{A}$ for $A(D)$ and $F_{B}$ for $B(D)$ respectively.

We introduce a generalized form of a theorem in Bochner and Martin [2] (see Theorem 1, Ch. V) as follows:

Proposition 5. Let $X$ be locally compact, $D$ be a subset of $X$ which is countable at $\infty$, and let $\bar{D}-D$ be first countable. Let $A=A(D)$ be a maximum modulus algebra and c.o. complete. Then $x \in F_{A}$ if and only if there is a function $f \in A$ such that $M_{f}(x)=\infty$. In fact, there is a single function $f$ such that $M_{f}(x)=\infty$ for all $x \in F_{A}$.

Proof. Use the analogous argument as in Bochner and Martin [2].

Theorem 3. Let $X$ be locally compact, $D$ be countable at $\infty$, and $\bar{D}-D$ be first countable. Let $A$ be a maximum modulus algebra and c.o. complete. Suppose $\Gamma_{B}$ is a minimal boundary and $F_{A}=\Gamma_{B}$ then every function $f \in A$ with $M_{f}\left(\Gamma_{B}\right)<\infty$ belongs to $B$.

Proof. Assume that $f$ is unbounded then there exists a sequence $\left\{x_{n}\right\} \subset D$ such that $\left|f\left(x_{n}\right)\right| \rightarrow \infty$ and $n \rightarrow \infty$. Let $x_{n} \rightarrow x$ then by Proposition 5, $x \in F_{A}$ and so $x \in \Gamma_{B}$. Thus $\infty=M_{f}(x) \leqq M_{f}\left(\Gamma_{B}\right)<\infty$, which is absurd. Hence $f \in B$.

We observe that $h \in S\left(B_{s}\right)-S\left(B_{c}\right)$ if and only if for any compact subset $K$ of $D$ there exists $f \in B$ ( $f$ may depend on $K$ ) such that $|h(f)|>\|f\|_{K}$.

THEOREM 4. Let $X$ be locally compact and $B$ separate the points of $D$. Let $F_{B}$ be the set of all frontier points for $B$. If $\rho D=S\left(B_{c}\right)$ then $\bar{D}-D=F_{B}$.

Proof. Let bdry $S\left(B_{c}\right)=\operatorname{cl} S\left(B_{c}\right)-S\left(B_{c}\right)$. By Proposition 3, $\pi\left(\right.$ bdry $\left.S\left(B_{c}\right)\right)=$ bdry $D$. Now if $h \in$ bdry $S\left(B_{c}\right)$, then for any com- 
pact subset $K$ of $D$, there exists $f \in B$ such that $|h(f)|>\|f\|_{K}$. We claim $M_{f}(\pi(h))>\|f\|_{K}$ : Suppose $M_{f}(\pi(h))=\|f\|_{K}=r$, then there exists a net $\left\{x_{n}\right\} \subset D$ such that ||$f\left(x_{n}\right)|-r|<1 / n$ as $x_{n} \rightarrow \pi(h)$. So $\left|f\left(x_{n}\right)\right| \rightarrow r$. Now, let $h_{x_{n}} \rightarrow h$. Since $\hat{f}$ is continuous, $\hat{f}\left(h_{x_{n}}\right) \rightarrow \hat{f}(h)$. So $f\left(x_{n}\right) \rightarrow h(f)$. In particular, $\left|f\left(x_{n}\right)\right| \rightarrow|h(f)|$. Then it follows that $|h(f)|=r=\|f\|_{K}$. This is absurd. Hence $M_{f}(\pi(h))>\|f\|_{K}$. So bdry $D=F_{B}$.

Note. If $D$ is a Stein manifold of bounded type then $\rho D=S\left(B_{c}\right)$ (see Kim [3]).

\section{REFERENCES}

1. F. Birtel, Uniform Algebras with Unbounded Functions, Rice studies, (1968), 1-13.

2. S. Bochner and W. Martin, Several Complex Variables, Princeton (1948).

3. Kim, Boundedly holomorphic convex domains, (to appear) Pacific J. Math., 45 (1973).

4. K. Hoffman, Bounded analytic functions and Gleason parts, Annals of Math., 86 (1967), 74-111.

5. F. Quigley, Generalized Phragmén-Lindelöf Theorems, Function algebras, the proceedings of the international conference on function algebras at Tulane, edited by $\mathrm{F}$. Birtel (1966), 36-41.

6. - Lectures on Several Complex Variables, Tulane Univ., 1963-1966.

7. C. Rickart, Boundary properties of sets relative to function algebras, Studia Math., 31 (1968), 253-261.

8. - General Theory of Banach Algebras, Princeton (1960).

Received November 10, 1971.

UNIVERSITY OF FLORIDA 


\section{PACIFIC JOURNAL OF MATHEMATICS}

EDITORS

\author{
H. SAMELSON \\ Stanford University \\ Stanford, California 94305 \\ C. R. HOBBY \\ University of Washington \\ Seattle, Washington 98105
}

\author{
J. DUGUNDJI \\ Department of Mathematics \\ University of Southern California \\ Los Angeles, California 90007 \\ RICHARD ARENS \\ University of California \\ Los Angeles, California 90024
}

\section{ASSOCIATE EDITORS}

E. F. BECKENBACH

B. H. NeUManN

F. WOLF

K. YosHIDA

\section{SUPPORTING INSTITUTIONS}

UNIVERSITY OF BRITISH COLUMBIA

CALIFORNIA INSTITUTE OF TECHNOLOGY

UNIVERSITY OF CALIFORNIA

MONTANA STATE UNIVERSITY

UNIVERSITY OF NEVADA

NEW MEXICO STATE UNIVERSITY

OREGON STATE UNIVERSITY

UNIVERSITY OF OREGON

OSAKA UNIVERSITY
UNIVERSITY OF SOUTHERN CALIFORNIA

STANFORD UNIVERSITY

UNIVERSITY OF TOKYO

UNIVERSITY OF UTAH

WASHINGTON STATE UNIVERSITY UNIVERSITY OF WASHINGTON

AMERICAN MATHEMATICAL SOCIETY NAVAL WEAPONS CENTER 


\section{Pacific Journal of Mathematics}

\section{Vol. 45, No. $1 \quad$ September, 1973}

William George Bade, Complementation problems for the Baire classes .......... 1

Ian Douglas Brown, Representation of finitely generated nilpotent groups ........ 13

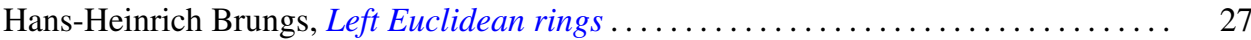

Victor P. Camillo and John Cozzens, A theorem on Noetherian hereditary rings ..... 35

James Cecil Cantrell, Codimension one embeddings of manifolds with locally flat

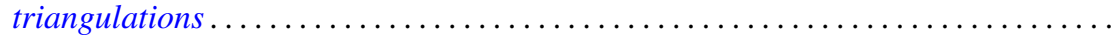

L. Carlitz, Enumeration of up-down permutations by number of rises . . . . . . . . . .

Thomas Ashland Chapman, Surgery and handle straightening in Hilbert cube

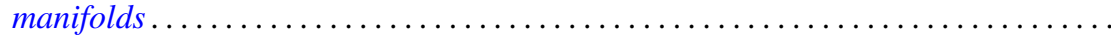

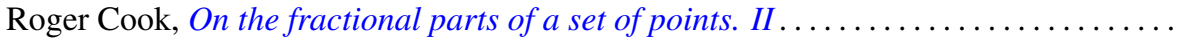

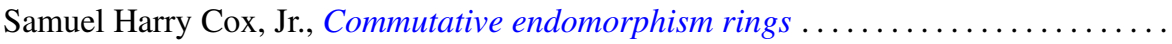

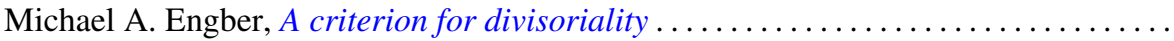

Carl Clifton Faith, When are proper cyclics injective . . . . . . . . . . . . . . 97

David Finkel, Local control and factorization of the focal subgroup . . . . . . . . . 113

Theodore William Gamelin and John Brady Garnett, Bounded approximation by

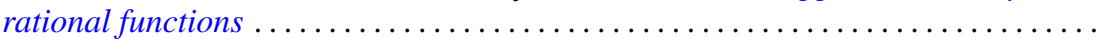

Kazimierz Goebel, On the minimal displacement of points under Lipschitzian

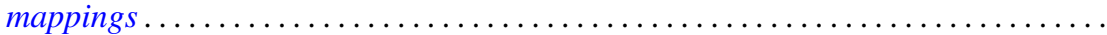

Frederick Paul Greenleaf and Martin Allen Moskowitz, Cyclic vectors for representations associated with positive definite measures: nonseparable

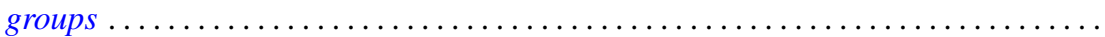

Thomas Guy Hallam and Nelson Onuchic, Asymptotic relations between perturbed linear systems of ordinary differential equations .

David Kent Harrison and Hoyt D. Warner, Infinite primes of fields and completions. .

James Michael Hornell, Divisorial complete intersections . ......

Jan W. Jaworowski, Equivariant extensions of maps ..............

John Jobe, Dendrites, dimension, and the inverse arc function .. .

Gerald William Johnson and David Lee Skoug, Feynman integrals of non-factorable

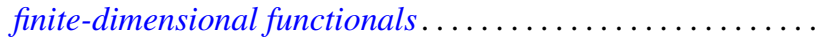

Dong S. Kim, A boundary for the algebras of bounded holomorphic functions ...... 269

Abel Klein, Renormalized products of the generalized free field and its derivatives ... 275

Joseph Michael Lambert, Simultaneous approximation and interpolation in $L_{1}$ and

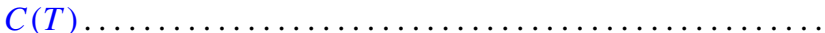

Kelly Denis McKennon, Multipliers of type $(p, p)$ and multipliers of the group

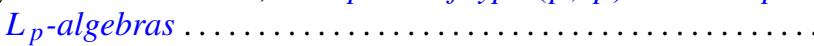

William Charles Nemitz and Thomas Paul Whaley, Varieties of implicative

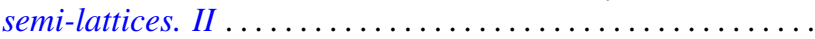

Donald Steven Passman, Some isolated subsets of infinite solvable

Norma Mary Piacun and Li Pi Su, Wallman compactifications on E-completely

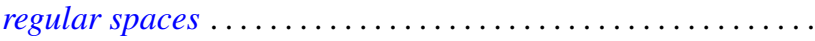

Jack Ray Porter and Charles I. Votaw, $S(\alpha)$ spaces and regular Hausdorff extensions....

Gary Sampson, Two-sided $L_{p}$ estimates of convolution transforms .

Ralph Edwin Showalter, Equations with operators forming a rig
Raymond Earl Smithson, Fixed points in partially ordered sets .

Victor Snaith and John James Ucci, Three remarks on symmetric products and

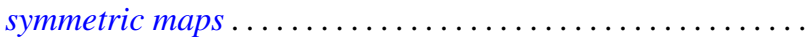

